\title{
A QUIMILUMINESCÊNCIA COMO FERRAMENTA ANALÍTICA: DO MECANISMO A APLICAÇÕES DA REAÇÃO DO LUMINOL EM MÉTODOS CINÉTICOS DE ANÁLISE
}

Ernesto Correa Ferreira e Adriana Vitorino Rossi*

Instituto de Química, Universidade Estadual de Campinas, CP 6154, 13083-970 Campinas - SP

Recebido em 18/4/01; aceito em 12/4/02

\begin{abstract}
CHEMILUMINESCENCE AS AN ANALYTICAL TOOL: FROM THE MECHANISM TO APPLICATIONS OF THE REACTION OF LUMINOL IN KINETIC BASED METHODS. Relevant aspects of proposed mechanisms of the chemiluminescent reaction of luminol are presented and commented to emphasize its perspectives for kinetic analysis. A careful search for analytical applications of this reaction is discussed in order to point out new trends of the studies. Kinetic analysis using the luminol reaction is proposed to be a very attractive due to the good performance of the reaction in analytical applications and the positive characteristics of kinetic analysis, such as low cost and sensibility. It is pointed out that kinetic analysis using the chemiluminescent reaction of luminol should be encouraged.
\end{abstract}

Keywords: luminol; chemiluminescence; kinetic analysis.

\section{QUIMILUMINESCÊNCIA}

\section{Breve histórico}

Desde a antiguidade, fenômenos de emissão de luz, como a quimiluminescência, eram descritos, mas, por muito tempo foram associados a mitos ou fantasmas. Em 1669, o médico H. Brandt, a partir da destilação exaustiva de uréia, produziu fósforo que, devido a sua oxidação pelo $\mathrm{O}_{2}$ do ar, produzia quimiluminescência ${ }^{1}$. O primeiro composto orgânico sintético luminescente descrito foi a lofina, 2,4,5-trifenilimidazol, obtida em 1887 por Radiziszewski ${ }^{2}$. A partir destes resultados, em 1888, Wiedemann conseguiu distinguir a quimiluminescência da incandescência, definindo-a como "a emissão de luz que ocorre junto a processos químicos"3. Desde esta época, as reações quimiluminescentes têm sido objeto de estudos para elucidação dos mecanismos envolvidos e da atuação de outras espécies que afetam a quimiluminescência. Além destes compostos exemplificados, outras substâncias como luminol, lucigenina, isoluminol, etanodioato de bis(2,4,6-triclorofenila), pirogalol e a luciferina também participam como substratos de reações quimiluminescentes e suas fórmulas são apresentadas na Figura $1^{4,5}$.

\section{QUIMILUMINESCÊNCIA E QUÍMICA ANALÍTICA}

A precisão e a sensibilidade das análises estão fortemente relacionadas com a técnica de medida e o sistema reacional empregados. Há grandes esforços em otimizar sistemas convencionais e encontrar novos sistemas, visando atender às novas e crescentes necessidades do mercado. Neste contexto, a quimiluminescência destaca-se por apresentar características de grande aplicabilidade analítica.

Quimiluminescência é a produção de radiação luminosa eletromagnética (inclusive ultravioleta ou infravermelho) por uma reação química. Quando esta radiação é emitida a partir de um sistema químico presente num organismo ou dele derivado, acaba sendo conhecida como bioluminescência ${ }^{4}$.

O processo químico da quimiluminescência envolve a absorção, pelos reagentes, de energia suficiente para geração de um complexo

*e-mail: adriana@iqm.unicamp.br<smiles>c1ccc(-c2nc(-c3ccccc3)c(-c3ccccc3)[nH]2)cc1</smiles>

1<smiles>Nc1ccc2c(=O)[nH][nH]c(=O)c2c1</smiles>

$\underline{4}$

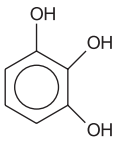<smiles>Nc1cccc2c(=O)[nH][nH]c(=O)c12</smiles>

$\underline{2}$
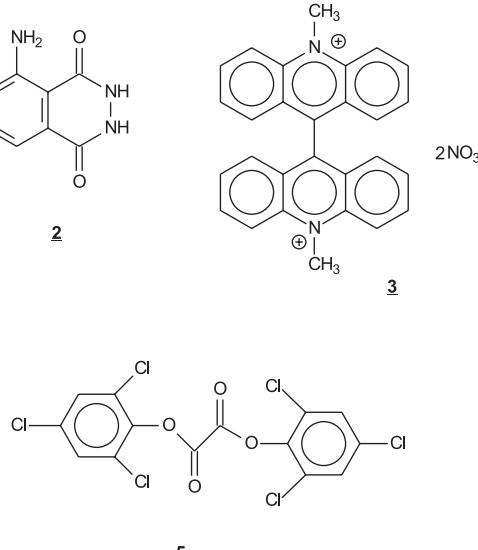

$\underline{5}$

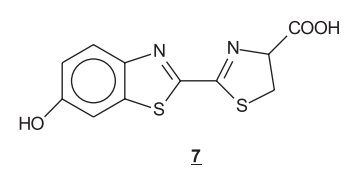

$\underline{7}$
Figura 1. Exemplos de compostos que participam como substratos de reações quimiluminescentes: $\underline{\mathbf{1}}$ lofina, $\underline{\mathbf{2}}$ luminol, $\underline{\mathbf{3}}$ lucigenina, $\underline{\mathbf{4}}$ isoluminol, $\underline{\mathbf{5}}$ etanodioato de bis(2,4,6-triclorofenila), $\underline{\mathbf{6}}$ pirogalol e $\underline{\mathbf{Z}}$ luciferina

ativado, o qual se transforma em um produto eletronicamente excitado $^{6}$. Se esta espécie excitada for emissiva, produz a radiação diretamente, caso contrário, pode ocorrer a transferência de energia do estado excitado formado para uma molécula aceptora apropriada, resultando na emissão indireta da radiação ${ }^{6}$. Pesquisas sobre reações quimiluminescente são desenvolvidas em todas as áreas tradicionais da Química e, em geral, envolvem estudos sobre mecanismos, identificação de reagentes, produtos e intermediários, além de medidas da eficiência quântica e desenvolvimento de aplicação analítica ${ }^{7}$.

As condições da reação têm um efeito significativo na quimiluminescência. $O$ tempo de reação e a duração da radiação emitida variam de períodos muito pequenos (menores que $1 \mathrm{~s}$ ) até 
muito longos (cerca de 1 dia). A mudança de parâmetros experimentais como forma de mistura dos reagentes, temperatura, concentrações de espécies reacionais ou interferentes, $\mathrm{pH}$, podem modificar totalmente a emissão, podendo até mesmo extingui-la ${ }^{5}$.

A intensidade da emissão depende da velocidade de reação, da eficiência na geração de moléculas em um estado excitado (expresso pelo rendimento quântico) e do fluoróforo, que pode ser considerado como a substância que produz a emissão $0^{1,8}$.

A cor e a intensidade de emissão também são influenciadas pela polaridade do solvente, pela temperatura do sistema e por outros processos físicos secundários de supressão da emissão da molécula no estado excitado ${ }^{5}$. Como exemplo, pode-se citar que o comprimento de onda de emissão máxima da reação quimiluminescente do luminol ( $\lambda_{\text {max }}$ de emissão) varia entre 431 e $500 \mathrm{~nm}$, de acordo com o solvente utilizado, conforme ilustra a Tabela $1^{8}$.

Tabela 1. $\lambda_{\max }$ de emissão da oxidação do luminol em diferentes solventes

\begin{tabular}{cc}
\hline solvente & $\lambda_{\max }$ de emissão $(\mathrm{nm})$ \\
\hline Água & 431 \\
Dimetilsulfóxido & 502 \\
Dimetilformamida & 499 \\
Acetonitrila & 500 \\
Tetra-hidrofurano & 496 \\
\hline
\end{tabular}

Como a intensidade de emissão é dependente da velocidade da reação, monitorá-la pode fornecer dados para quantificar qualquer espécie cuja concentração afete esta velocidade ${ }^{5}$.

As principais características das reações quimiluminescentes adequadas para aplicação analítica são os excelentes limites de detecção que podem ser alcançados e a simplicidade da instrumentação necessária para registrar o sinal analítico ${ }^{5}$. Com reações bioluminescentes, pode-se trabalhar em condições de especificidade quando envolvem catálise enzimática.

A elevada sensibilidade de métodos analíticos com reações quimiluminescentes, que serão identificados neste texto com técnicas de quimiluminescência, está ligada a vários fatores, como a ausência da necessidade de uma fonte de radiação, o que diminui ou elimina os espalhamentos Raman e Rayleigh, e o ruído associado com este tipo de componente, aumentando a razão sinal/ruído da medida. Limites de detecção na ordem de fentomol $\left(10^{-15} \mathrm{~mol}\right)$ não são incomuns nas técnicas de quimiluminescência e em alguns sistemas enzimáticos já foram detectadas cerca de 120 moléculas de analito ${ }^{5}$.

As reações quimiluminescentes podem ocorrer tanto em fase gasosa como também nas fases sólidas e líquidas. As principais reações em fase gasosa já estudadas são aquelas que ocorrem entre o ozônio e óxidos de nitrogênio e são utilizadas para a determinação de ozônio na atmosfera ${ }^{8}$. Em cromatografia gasosa, vários sistemas envolvendo reações quimiluminescentes já foram citados para a detecção de compostos contendo $\mathrm{N}$ e $\mathrm{S}$. Porém, segundo Worsfold $^{10}$, a fase líquida apresenta as maiores perspectivas para aplicação analítica da quimiluminescência, tanto em meio aquoso como não-aquoso.

Em solução, a quimiluminescência pode ser empregada em várias aplicações analíticas para determinação de íons metálicos, ânions inorgânicos, biomoléculas, substâncias carcinogênicas e drogas em diferentes matrizes ambientais e clínicas ${ }^{5,10}$. Dentre os diferentes tipos de espécies analisadas por esta técnica, o controle de compostos orgânicos no meio ambiente, como pesticidas ${ }^{11}$ e compostos de $\mathrm{S}$ derivados do petróleo ${ }^{12}$, são exemplos de destaque pela grande sensibilidade e simplicidade.
Uma reação quimiluminescente de grande importância envolve a oxidação da luciferina, um dos compostos responsáveis pela luz do vagalume, em presença de ATP e é utilizada para a determinação de biomassa e da presença de vida em corpos estrelares, além de servir como forma de detecção em vários imunoensaios ${ }^{10}$. Outra reação, das mais aplicadas em métodos analíticos, é a famosa oxidação do luminol em meio alcalino ${ }^{13}$.

\section{A REAÇÃO QUIMILUMINESCENTE DO LUMINOL}

Data de 1928, o trabalho pioneiro sobre a reação do luminol, publicado por Albrecht ${ }^{14}$. A oxidação do luminol por $\mathrm{H}_{2} \mathrm{O}_{2}$ em meio alcalino foi a primeira reação quimiluminescente descrita e suas propriedades termodinâmicas e cinéticas continuam sendo intensamente estudadas até os dias de hoje. Várias propostas mecanísticas para a reação já foram apresentadas e algumas etapas já estão quase completamente estabelecidas ${ }^{4,5,13}$. Restam, ainda, aspectos não totalmente elucidados, envolvendo a formação de estados eletronicamente excitados que, como na maioria das reações quimiluminescentes, ainda não estão bem esclarecidos e continuam sendo objeto de pesquisas e polêmicas recentes ${ }^{4,15}$. As idéias apresentadas na seqüência não têm a pretensão de esgotar o tema nem cobrir todas as obras já publicadas sobre o assunto mas servem para ilustrar alguns aspectos da reação.

Vários pesquisadores já citaram alguns caminhos possíveis para a reação do luminol com alguns agentes oxidantes, como $\mathrm{O}_{2}$ e $\mathrm{H}_{2} \mathrm{O}_{2}$. Também foram determinados alguns possíveis intermediários. Além disso, estudos realizados com outras reações quimiluminescentes (por exemplo, com dioxetanos) têm mostrado novos caminhos e idéias sobre o mecanismo de quimiluminescência ${ }^{7}$. Uma interessante proposta mecanística foi apresentada por Albertin e colaboradores ${ }^{4}$, sendo apresentada na Figura 2.
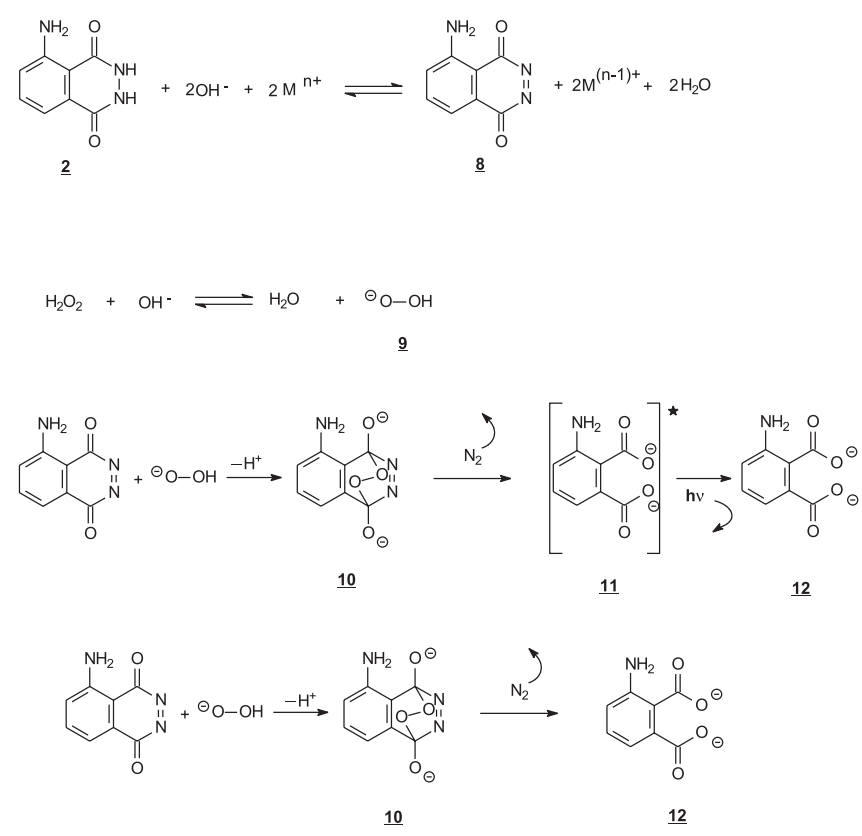

Figura 2. Proposta mecanística de Albertin e colaboradores ${ }^{4}$ para a reação quimiluminescente do luminol em meio alcalino e na presença de um íon de metal de transição $\left(\mathrm{M}^{n+}\right)$, utilizando o $\mathrm{H}_{2} \mathrm{O}_{2}$ como agente oxidante

Nesta proposta, a etapa para a emissão da luminescência está ligada ao aparecimento da forma eletronicamente excitada do 3amino-ftalato 11. O aparecimento desta espécie está ligada às condi- 
ções do meio reacional, como pH, concentração dos reagentes e composição do tampão. O produto final da reação $\underline{\mathbf{1 2}}$ pode ser formado diretamente sem luminescência ou pela "relaxação" da espécie eletronicamente excitada 11 com luminescência. Há importantes etapas intermediárias, como a formação da diazoquinona $\underline{\mathbf{8}}$ a partir do luminol e a formação do ânion de $\mathrm{H}_{2} \mathrm{O}_{2} \underline{\text { 9 }}$, que estabelecem relações entre as espécies envolvidas na formação do intermediário endoperóxido $\underline{\mathbf{1 0}}$, cuja decomposição produz ou não a quimiluminescência.

Com toda a complexidade da reação, é pertinente destacar algumas descobertas significativas sobre os intermediários e as etapas da reação quimiluminescente do luminol.

\section{Detalhes mecanísticos da reação do luminol}

Na década de 80 , Merényi e colaboradores ${ }^{15-22}$ avançaram na descoberta de algumas etapas do mecanismo da reação, utilizando a técnica de radiólise e modelos computacionais. Foram verificados e confirmados alguns intermediários, como a diazoquinona $\underline{\mathbf{8}}$ e a produção da luminescência pelo 3 -aminoftalato excitado $\underline{\mathbf{1 1}}$, a partir da reação com o radical $\mathrm{ClO}_{2}$ - formado pela radiólise do dióxido de

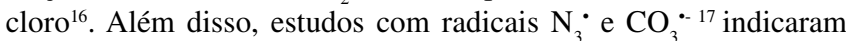
novos possíveis intermediários na formação e decomposição do endoperóxido $\underline{\mathbf{1 0}}$, como o radical de luminol $\underline{13}$ e o a-hidroxihidroperóxido $\underline{14}$, Figura 3.

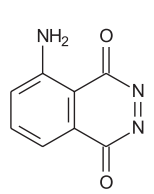

$\underline{8}$

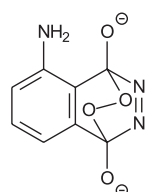

$\underline{10}$

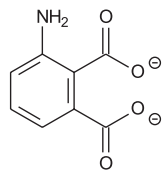

$\underline{12}$<smiles>Nc1cccc2c(=O)[nH][nH]c(=O)c12</smiles>

$\underline{13}$

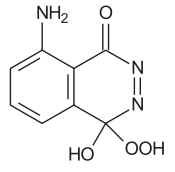

$\underline{14}$
Figura 3. Estruturas de alguns intermediários da reação quimiluminescente do luminol: $\underline{8}$ diazoquinona, $\underline{10}$ endo-peróxido, $\underline{12}$ diânion do ácido ftálico, $\underline{13}$ radical do luminol e $\underline{14}$ hidro-peróxido ${ }^{15-22}$

Parâmetros como constantes de velocidade, dependência do $\mathrm{pH}$ reacional e efeitos da participação de outras espécies (cátions metálicos e ânions como carbonato e brometo) no aumento da luminescência, também foram discutidos em propostas mecanísticas ${ }^{18-20}$. Além disso, modelagem computacional foi empregada para investigação da existência e da decomposição do intermediário endo-peróxido do luminol ${\underline{\mathbf{1 0}^{21}}}^{\text {. }}$.

A emissão de radiação pela reação de oxidação de luminol está ligada à formação do produto eletronicamente excitado $\underline{11}$, que é formado a partir da decomposição do endo-peróxido $\underline{\mathbf{1 0}}$. Segundo a proposta de $\mathrm{Michl}^{23}$, a decomposição do endo-peróxido $\underline{\mathbf{1 0}}$ ocorre quando a ligação $\sigma$ entre os dois átomos de oxigênio se rompe, podendo ou não formar o produto excitado. No caso do produto excitado, possivelmente, não há tempo suficiente para os elétrons se distribuírem entre os novos orbitais, deixando o novo orbital LUMO ainda ocupado, como mostrado na Figura 4. Por este raciocínio, podese supor que os caminhos de formação de produto excitado e não excitado possuem, em algum momento, energias semelhantes. Poderia haver um "cruzamento" entre esses caminhos, levando à formação do produto excitado, conforme o diagrama da Figura 5.

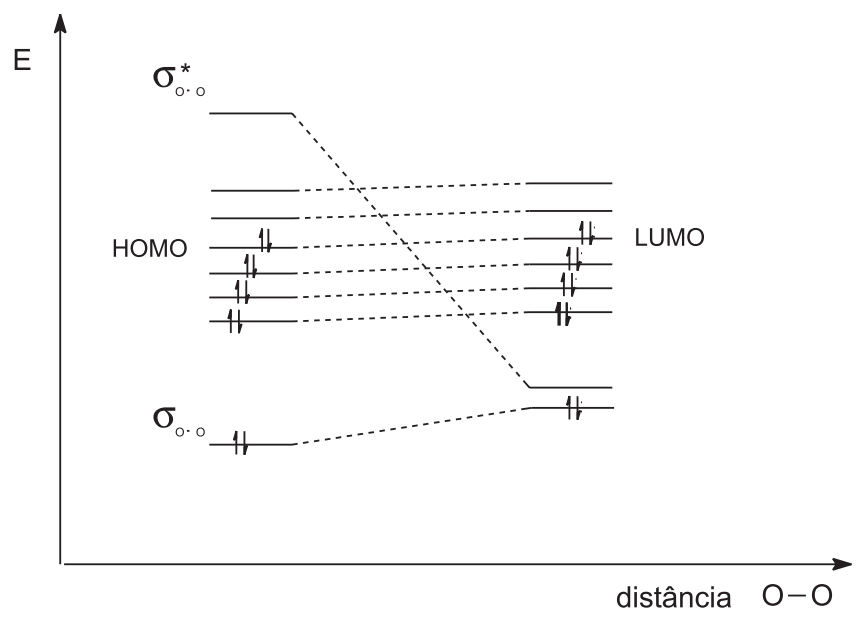

Figura 4. Diagrama esquemático dos orbitais moleculares do sistema endoperóxido $\underline{10}$ / 3-amino-ftalato $\underline{12}$

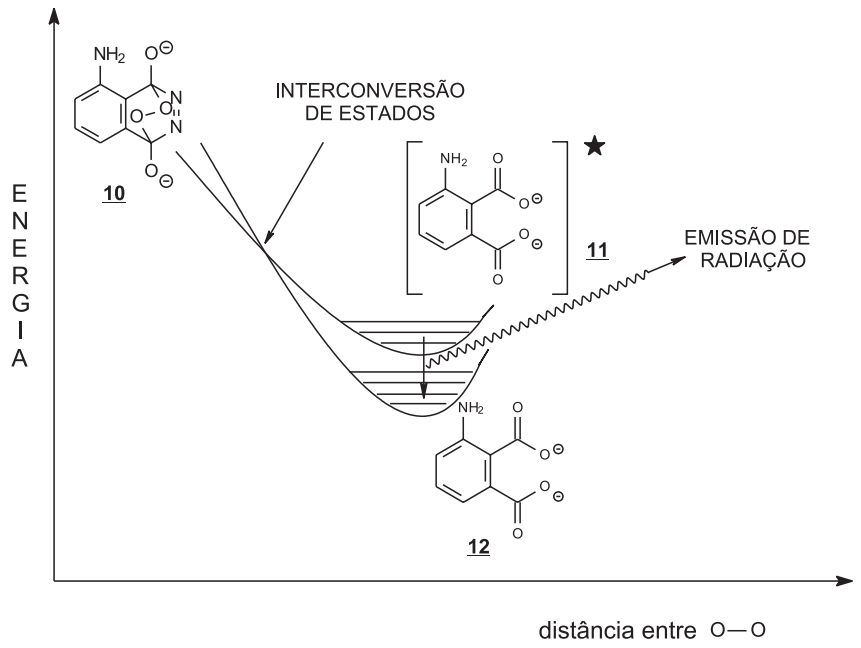

Figura 5. Diagrama esquemático dos estados do endo-peróxido 10 / 3 aminoftalato $\underline{12}$, relacionando a energia do sistema com o rompimento da ligação dos átomos de oxigênio no intermediário eletronicamente excitado $\underline{11}$

Porém, o intermediário endo-peróxido ainda não foi detectado. Isto pode ser justificado por uma suposta elevadíssima velocidade de decomposição, que inviabilizaria sua detecção.

Todos estes estudos possibilitaram descobertas de alguns detalhes mecanísticos que, eventualmente, poderão conduzir à elucidação completa da reação. Nas últimas décadas, Merényi e colaboradores ${ }^{15}$ alcançaram grandes avanços na resolução deste problema mecanístico, sendo as conclusões resumidos e reinterpretadas em trabalho publicado no Journal of Bioluminescence and Chemiluminescence ${ }^{15}$. Segundo estes autores, o processo de oxidação pode ser dividido em duas etapas: (i) os caminhos que levam ao intermediário principal, identificado como $\alpha$-hidroxi-hidroperóxido $\underline{\mathbf{1 4}}$; e (ii) a decomposição deste intermediário (conforme ilustrado na Figura 6). A primeira etapa é significativamente dependente da composição do meio reacional, como $\mathrm{pH}$, as concentrações dos reagentes, a natureza dos agentes oxidantes, a composição do tampão e a presença de qualquer outra espécie que interfira nestas interações. Por outro lado, a decomposição do $\alpha$-hidroxi-hidroperóxido $\underline{\mathbf{1 4}}$ parece depender apenas do $\mathrm{pH}$ do meio ${ }^{15}$. A Figura 6 apresenta um esquema condensado da proposta mecanística da reação de oxidação de luminol ${ }^{15}$, onde as 


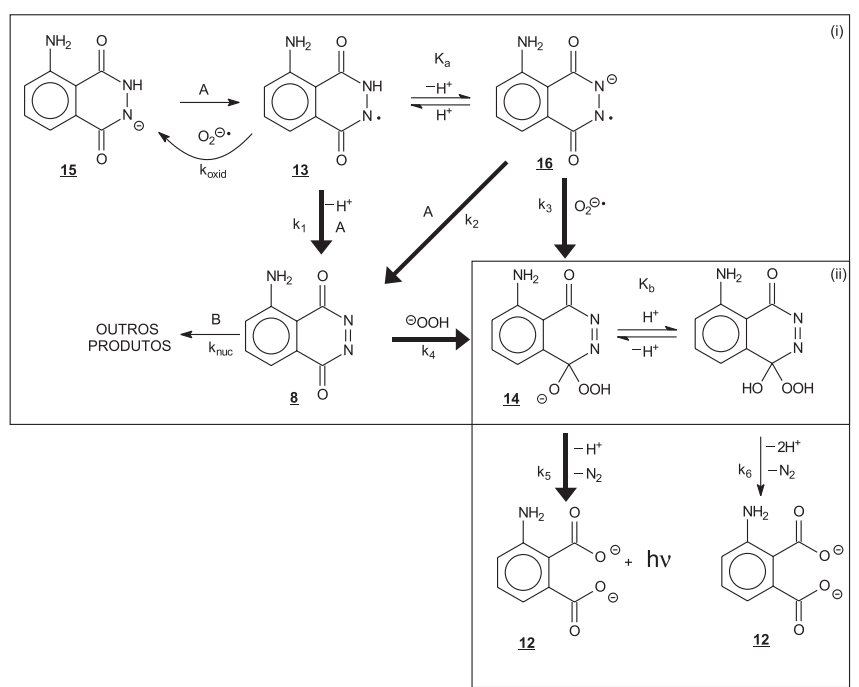

Figura 6. Esquema simplificado do mecanismo de oxidação do luminol. A representa espécies aceptoras de 1 elétron e $\boldsymbol{B}$ representa nucleófilos. Os valores das constantes cinéticas e de equilíbrio das diversas etapas estão apresentados na Tabela 2

setas alargadas foram usadas para destacar os caminhos que levam à formação do intermediário $\alpha$-hidroxi-hidroperóxido $\underline{14}$, responsável direto pela produção da quimiluminescência. É de grande pertinência aos interessados na aplicação desta reação, alcançar condições que suprimam as reações laterais que não levam à quimiluminescência ${ }^{15}$. A Tabela 2 traz as constantes cinéticas e de equilíbrio envolvidas.

Pela proposta da Figura 6, inicialmente, o monoânion do luminol 15 é oxidado por uma espécie aceptora de um elétron, $\mathbf{A}$, formando o radical do luminol $\underline{\mathbf{1 3}}$ que estabelece um equilíbrio com sua espécie desprotonada 16. $\mathrm{O}$ aceptor $\mathbf{A}$ pode ser qualquer espécie que possa "receber" um elétron, como os radicais do luminol $\underline{\mathbf{1 3}}$ e $\underline{\mathbf{1 6}}$, os radicais $\mathrm{OH}^{*}, \mathrm{O}_{2} \cdot{ }^{\bullet}$ e $\mathrm{O}_{2}$, e cátions de metais de transição e vários de seus complexos, como ftalocianinas e proteínas. A velocidade de formação do radical de luminol $\underline{\mathbf{1 3}}$ depende da composição de $\mathbf{A}$, variando para cada sistema particular. Como esta etapa determina a velocidade da reação, sua cinética pode ser determinada pelo decaimento da quimiluminescência ${ }^{15}$. O papel do radical de luminol

Tabela 2. Valores das constantes cinéticas e de equilíbrio da Figura 6

\begin{tabular}{cccc}
\hline Constante & Valor & Unidade & Referência \\
\hline $\mathrm{k}_{\text {oxid }}$ & $7,1 \times 10^{9}$ & $\mathrm{~L} \mathrm{~mol}^{-1} \mathrm{~s}^{-1}$ & 18,19 \\
$2 \mathrm{k}_{1}(\mathbf{A}=\underline{\mathbf{1 3}})$ & $1,8 \times 10^{9}$ & $\mathrm{~L} \mathrm{~mol}^{-1} \mathrm{~s}^{-1}$ & 22 \\
$2 \mathrm{k}_{2}(\mathbf{A}=\underline{\mathbf{1 6}})$ & $5,0 \times 10^{8}$ & $\mathrm{~L} \mathrm{~mol}^{-1} \mathrm{~s}^{-1}$ & 22 \\
$\mathrm{k}_{2}\left(\mathbf{A}=\mathrm{O}_{2}\right)$ & $5,5 \times 10^{2}$ & $\mathrm{~L} \mathrm{~mol}^{-1} \mathrm{~s}^{-1}$ & 18 \\
$\mathrm{k}_{3}$ & $2,3 \times 10^{8}$ & $\mathrm{~L} \mathrm{~mol}^{-1} \mathrm{~s}^{-1}$ & 18 \\
$\mathrm{k}_{4}$ & $5,0 \times 10^{7}$ & $\mathrm{~L} \mathrm{~mol}^{-1} \mathrm{~s}^{-1}$ & 19 \\
$\mathrm{k}_{5}$ & $2,5 \times 10^{5}$ & $\mathrm{~s}^{-1}$ & 19 \\
$\mathrm{k}_{6}$ & $1,5 \times 10^{3}$ & $\mathrm{~s}^{-1}$ & 19 \\
$\mathrm{k}_{\text {nuc }}\left(\mathbf{B}=\mathrm{OH}^{-}\right)$ & $4,0 \times 10^{6}$ & $\mathrm{~L} \mathrm{~mol}^{-1} \mathrm{~s}^{-1}$ & 19 \\
$\mathrm{k}_{\text {nuc }}\left(\mathbf{B}=\mathrm{H}_{2} \mathrm{O}\right)$ & 0,45 & $\mathrm{~L} \mathrm{~mol}^{-1} \mathrm{~s}^{-1}$ & 19 \\
$\mathrm{k}_{\text {nuc }}\left(\mathbf{B}=\mathrm{H}_{2} \mathrm{O}_{2}\right)$ & $1,1 \times 10^{2}$ & $\mathrm{~L} \mathrm{~mol}^{-1} \mathrm{~s}^{-1}$ & 19 \\
$\mathrm{k}_{\text {nuc }}(\mathbf{B}=\underline{\mathbf{1 5}})$ & $1,3 \times 10^{4}$ & $\mathrm{~L} \mathrm{~mol}^{-1} \mathrm{~s}^{-1}$ & 19 \\
$\mathrm{~K}_{\mathrm{a}}$ & $2 \times 10^{-8}$ & $\mathrm{~mol} \mathrm{~L}^{-1}$ & 19 \\
$\mathrm{~K}_{\mathrm{b}}$ & $4 \times 10^{-11}$ & $\mathrm{~mol} \mathrm{~L}^{-1}$ & 19 \\
\hline
\end{tabular}

$\underline{13}$ depende da concentração do radical superperóxido, $\mathrm{O}_{2}^{-}$, gerado lentamente pela reação entre $\underline{13}$ e $\mathrm{O}_{2}$. Assim, no início da reação, o meio estaria na ausência de $\mathrm{O}_{2}^{\cdot}$ e os caminhos levariam à formação de diazoquinona $\underline{\mathbf{8}}$, o que resultaria na formação destas duas espécies no meio reacional ${ }^{15}$. A preferência pelo caminho que formará o $\alpha$-hidroxi-hidroperóxido $\underline{\mathbf{1 4}}$ será regida pela (i) concentração de $\mathrm{O}_{2}$ no meio, cuja presença favorece a formação do superperóxido; ou (ii) pela concentração de $\mathrm{H}_{2} \mathrm{O}_{2}$ no meio, que aumenta a velocidade da reação entre $\mathrm{HO}_{2}^{-}$e a diazoquinona $\underline{\mathbf{8}}$. Além disso, a radiação emitida pelo caminho do superperóxido tem um ótimo em $\mathrm{pH}$ 9,2, decorrente da reação diferenciada entre este radical e os radicais $\underline{\mathbf{1 3}}$ e 16. Por outro lado, a radiação emitida pelo caminho da diazoquinona $\underline{\mathbf{8}}$ é regida pela concentração de $\mathrm{H}_{2} \mathrm{O}_{2}$ em relação a outros nucleófilos presentes. Assim, se a concentração de $\mathrm{H}_{2} \mathrm{O}_{2}$ for suficientemente alta para competir com superperóxido, a diazoquinona $\underline{\mathbf{8}}$ é convertida quantitativamente no $\alpha$-hidroxi-hidroperóxido $\underline{\mathbf{1 4}}$ independente do $\mathrm{pH}$ do meio ${ }^{15}$.

A maioria das aplicações desta reação para a análise de cátions de metais de transição (Ni(II), Th, Tl, As, Al(III), Sn(II), Pb(II), Bi(III), $\mathrm{Cr}(\mathrm{III}), \mathrm{Fe}(\mathrm{II}), \mathrm{Fe}(\mathrm{III}), \mathrm{Os}(\mathrm{III}), \mathrm{Co}(\mathrm{II}), \mathrm{Cu}(\mathrm{II}), \mathrm{Zn}$ (II), $\mathrm{Pd}(\mathrm{II}), \mathrm{Ag}(\mathrm{I})$, $\mathrm{Au}(\mathrm{III}), \mathrm{Hg}(\mathrm{II}), \mathrm{Ce}(\mathrm{IV}), \mathrm{U}(\mathrm{VI}), \mathrm{Sb}$ (III), Zr (IV), V(III), Mn(II), e $\mathrm{Cd}(\mathrm{II}))^{5}$ traz a idéia de que estes íons apresentam ação catalítica nesta reação, pelo efeito significativo que pequenas concentrações destes cátions promovem na velocidade da reação, o que caracteriza a elevada sensibilidade, típica de métodos cinéticos catalíticos ${ }^{13,24,25}$. Contudo, dentre os pesquisadores do mecanismo da reação ainda há controvérsias sobre real papel destes cátions no mecanismo da reação.

Xiao e colaboradores ${ }^{24}$ propuseram que estes cátions metálicos poderiam tanto interagir com o luminol e seus intermediários quanto com o $\mathrm{H}_{2} \mathrm{O}_{2}$, cuja decomposição produziria o radical $\mathrm{OH}^{*}$. Assim, estes autores apresentam 3 propostas para o efeito destes cátions: (i) a ativação da etapa de produção de radicais do $\mathrm{H}_{2} \mathrm{O}_{2}^{26,27}$; (ii) a formação do $\alpha$-hidroxi-hidroperóxido $\underline{14}$; e/ou (iii) a decomposição do $\alpha$ hidroxi-hidroperóxido $\underline{\mathbf{1 4}}^{24}$. Embora suas propostas estejam fundamentadas em resultados empíricos, a complexidade do mecanismo desta reação torna recomendável mais detalhamento experimental, o que pode ser encontrado nos trabalhos de Merényi e colaboradores $^{15-22}$, focados na elucidação mecanística e não numa aplicação analítica, como no caso do grupo de Xiao.

Na proposta (i) de Xiao e colaboradores, os cátions dos metais e seus complexos atuariam na decomposição catalítica do $\mathrm{H}_{2} \mathrm{O}_{2}$, produzindo o radical $\mathrm{OH}^{*}$ que é muito reativo com o luminol e seus intermediários. Contudo, cerca de $50 \%$ destas reações com $\mathrm{OH}^{*}$ produzem compostos que não levam à produção da espécie $\underline{\mathbf{1 4}}^{15}$, que seria o principal atuante na quimiluminescência. Porém, isto não considera que a decomposição do $\mathrm{H}_{2} \mathrm{O}_{2}$ forma outros radicais, como o superperóxido, $\mathrm{O}_{2}^{-}$, cuja reação com o radical luminol desprotonado $\underline{16}$ leva diretamente a formação de $\underline{\mathbf{1 4}} \mathrm{e}$, conseqüentemente, à quimiluminescência.

Na proposta (ii), a atuação dos cátions levaria à formação de $\underline{\mathbf{1 4}}$ o que, pela proposta de Merényi e colaboradores, é coerente, pois alguns cátions podem funcionar como um receptor de 1 elétron do monoânion do luminol $\underline{15}$ e do radical de luminol $\underline{13}$, formando a diazoquinona $\underline{\mathbf{8}}$ cuja reação com $\mathrm{HO}_{2}$ - forma $\underline{\mathbf{1 4}}$ e leva à quimiluminescência.

A proposta (iii), sugere que a decomposição de $\underline{14}$ seria catalisada pelos cátions metálicos, que não concorda com a proposta mecanística elaborada por Merényi e colaboradores ${ }^{15}$, que limita esta etapa do mecanismo exclusivamente ao efeito do $\mathrm{pH}$ do meio ${ }^{15}$. Neste contexto, a suposta ação "catalítica" dos cátions possivelmente seria um equívoco, já que os mesmos atuam tanto na produção de radicais do $\mathrm{H}_{2} \mathrm{O}_{2}$ quanto na produção dos intermediários $\underline{\mathbf{1 3}}$ e $\underline{\mathbf{8}}$. 
Como uma pequena variação de concentração dos cátions metálicos produz variações significativas na luminescência produzida, pode-se supor que este efeito tenha sido relacionado com a ação catalítica dos cátions nos trabalhos de aplicação analítica da reação, como pode ser notado na classificação de Motolla ${ }^{13}$. Contudo, a confirmação desta ação catalítica ainda carece de estudos mais elaborados como, por exemplo, estudos cinéticos simultâneos das atuações das espécies presentes neste sistema reacional.

É fato que a presença de íons $\mathrm{CO}_{3}{ }^{2-} \mathrm{e} \mathrm{Br}{ }^{-}$na reação proporciona o aumento da luminescência observada, pois estas espécies participam do mecanismo da reação. A interação entre os radicais $\mathrm{OH}^{*}$ e o luminol pode gerar outros produtos, além do radical do luminol $\underline{\mathbf{1 3}}$. Os radicais $\mathrm{OH}^{\cdot}$ podem reagir com luminol em posições do anel aromático e não produzir o $\alpha$-hidroxi-hidroperóxido $\underline{14}$ nem a quimiluminescência. Na presença dos íons $\mathrm{CO}_{3}^{2-}$ e $\mathrm{Br}$, os radicais $\mathrm{OH}^{*}$ são convertidos a radicais $\mathrm{CO}_{3}{ }^{-}$ou $\mathrm{Br}{ }^{*}$, que reagem seletivamente com o luminol, produzindo o radical do luminol $\underline{13}$ e favorecendo a produção de luminescência ${ }^{24}$. Efeitos análogos foram observados em estudos de aplicação da quimiluminescência do luminol para determinação de ácido ascórbico, que é um modificador da velocidade da reação ${ }^{28,29}$.

Parece ser consensual que, dependendo do sistema utilizado para o monitoramento (forma de adição e mistura de reagentes e sistema de detecção) e da procedência e tempo de utilização dos reagentes, os resultados quantitativos e, às vezes qualitativos, podem variar ${ }^{24}$. Geralmente, esta variação de resultados está ligada à velocidade de resposta do sistema de detecção e à forma de preparação e mistura dos reagentes, já que existem vários fatores cinéticos que modificam as concentrações das espécies responsáveis pela intensidade e duração da quimiluminescência.

A despeito de alguma indefinição sobre detalhes do mecanismo, a reação quimiluminescente do luminol apresenta grande potencialidade para aplicações analíticas. Isto pode ser verificado pelo grande número de trabalhos publicados nos últimos anos, conforme apresentado na sequiência.

\section{Aplicações analíticas da quimiluminescência do luminol}

As aplicações da reação quimiluminescente do luminol em Química Analítica podem ser divididas de acordo com a participação do analito na reação. Em aplicações diretas, o analito é uma espécie que participa da reação do luminol como reagente, "catalisador" ou modificador. Já em aplicações indiretas, o analito envolve a geração ou o consumo de espécies que participam da reação de luminol, sendo esta utilizada como a forma de detecção de outras reações ou técnicas, como em alguns métodos de separação e em imunoensaios.

Dentre as aplicações indiretas, destaca-se a utilização da reação na determinação de $\mathrm{H}_{2} \mathrm{O}_{2}$ produzido pela oxidação da espécie de interesse por enzimas e os biossensores. Nestes casos, além de elevada sensibilidade, obtém-se seletividade, empregando-se, geralmente, enzima imobilizada em fase sólida. Com estas técnicas foram já analisados substratos de enzimas oxidases, como amino-ácidos ${ }^{30}$, metanol $^{31}$, açúcares e DNA ${ }^{32}$.

Nas aplicações diretas, as determinações cinéticas (metais de transição) e as determinações de inibidores (compostos orgânicos e metais de transição) destacam-se em aplicações bastante precisas e com baixos limites de detecção.

Os metais de transição apresentam efeitos catalíticos na reação de oxidação do luminol, porém, em alguns casos, a mistura de alguns cátions inibe a reação. Este comportamento já foi descrito em vários trabalhos ${ }^{5,7}$. Mais de 30 íons metálicos inibem ou catalisam esta reação, sendo que o limite de detecção para alguns deles chega a $10^{-11} \mathrm{~mol} \mathrm{~L}^{-1}$. Compostos orgânicos que, em geral, atuam como inibidores da luminescência da reação, têm sido analisados em estudos que caracterizam as propostas de aplicação mais promissoras. A Tabela 3 traz informações sobre alguns sistemas já estudados ${ }^{34-72}$.

Além disso, a análise de misturas de metais ou de compostos orgânicos por esta reação, com obtenção de dados multidimensionais, é considerada uma ferramenta analítica versátil, precisa e sensível ${ }^{73}$, que merece ser mais estudada.

Estatisticamente, pode-se verificar o crescimento atual do número de aplicações analíticas do luminol, como apresentado na Figura 7. As informações apresentadas na seqüência foram obtidas num levantamento bibliográfico no banco de dados eletrônico WEB OF $\mathrm{SCIENCE}^{74}$, com as seguintes palavras chaves: "luminol and (determination or analysis)".

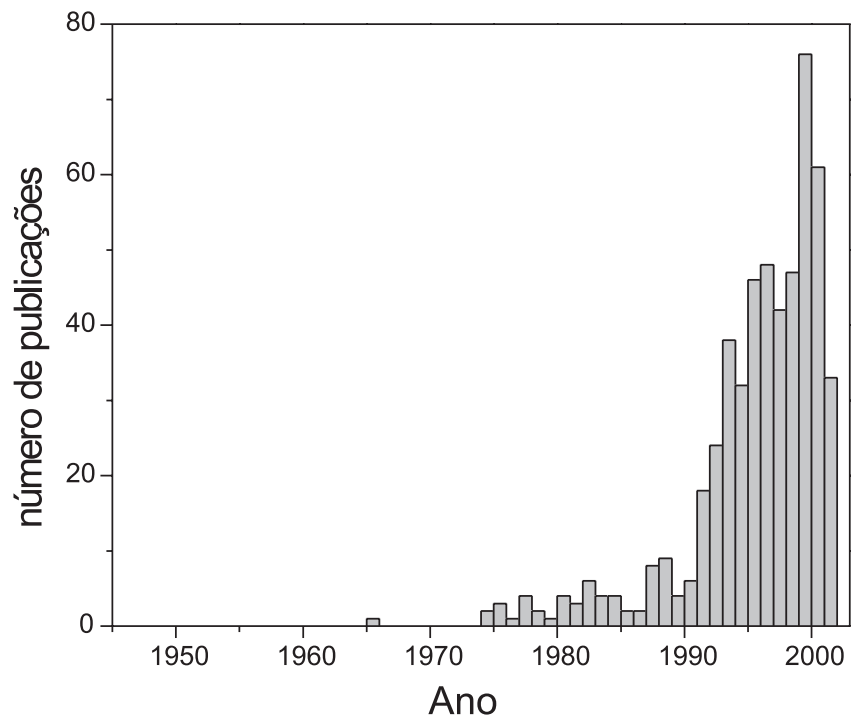

Figura 7. Publicações sobre a reação quimiluminescente do luminol como aplicação analítica desde 1945

As primeiras publicações de métodos analíticos com a reação do luminol aparecem a partir de 1965, tendo como principais contribuintes no seu desenvolvimento o Japão, os EUA, a China e a Espanha, com maior número de trabalhos nesta linha de pesquisa.

A detecção de intensidade de quimiluminescência vem sendo aplicada em várias técnicas analíticas consagradas. Avaliando-se as publicações destacadas pela pesquisa bibliográfica realizada, verificou-se que as técnicas que mais utilizam a quimiluminescência do luminol são os métodos de análise por injeção em fluxo, inclusive com reagente imobilizado, os métodos de separação (cromatografia líquida de alta eficiência, cromatografia líquida, cromatografia gasosa e eletroforese) e os métodos de imunoensaios. Dentre todos os trabalhos encontrados, deve ser salientado que apenas $6 \%$ envolvem métodos cinéticos. Outras técnicas que podem ser destacadas são o monitoramento estático de quimiluminescência, biossensores e eletroquimiluminescência, como é apresentado na Figura 8.

Vale destacar o tipo de matriz e analito mais comumente analisados com métodos que envolvem reações quimiluminescentes. A maioria dos trabalhos publicados aplica-se a amostras de fluídos biológicos, como tecidos humanos e de animais, além de excreções (urina) e sangue (plasma e soro sanguíneo), com ênfase em analitos orgânicos como glicose ${ }^{32}$, colesterol e cortisona ${ }^{59}$.

Neste contexto, verifica-se que a técnica de detecção de intensidade da quimiluminescência da reação do luminol apresenta aplicabilidade reconhecida para análise de diversos compostos orgânicos, em vários tipos de amostras, principalmente de origem bioló- 
Tabela 3. Algumas aplicações analíticas com a reação quimiluminescente do luminol

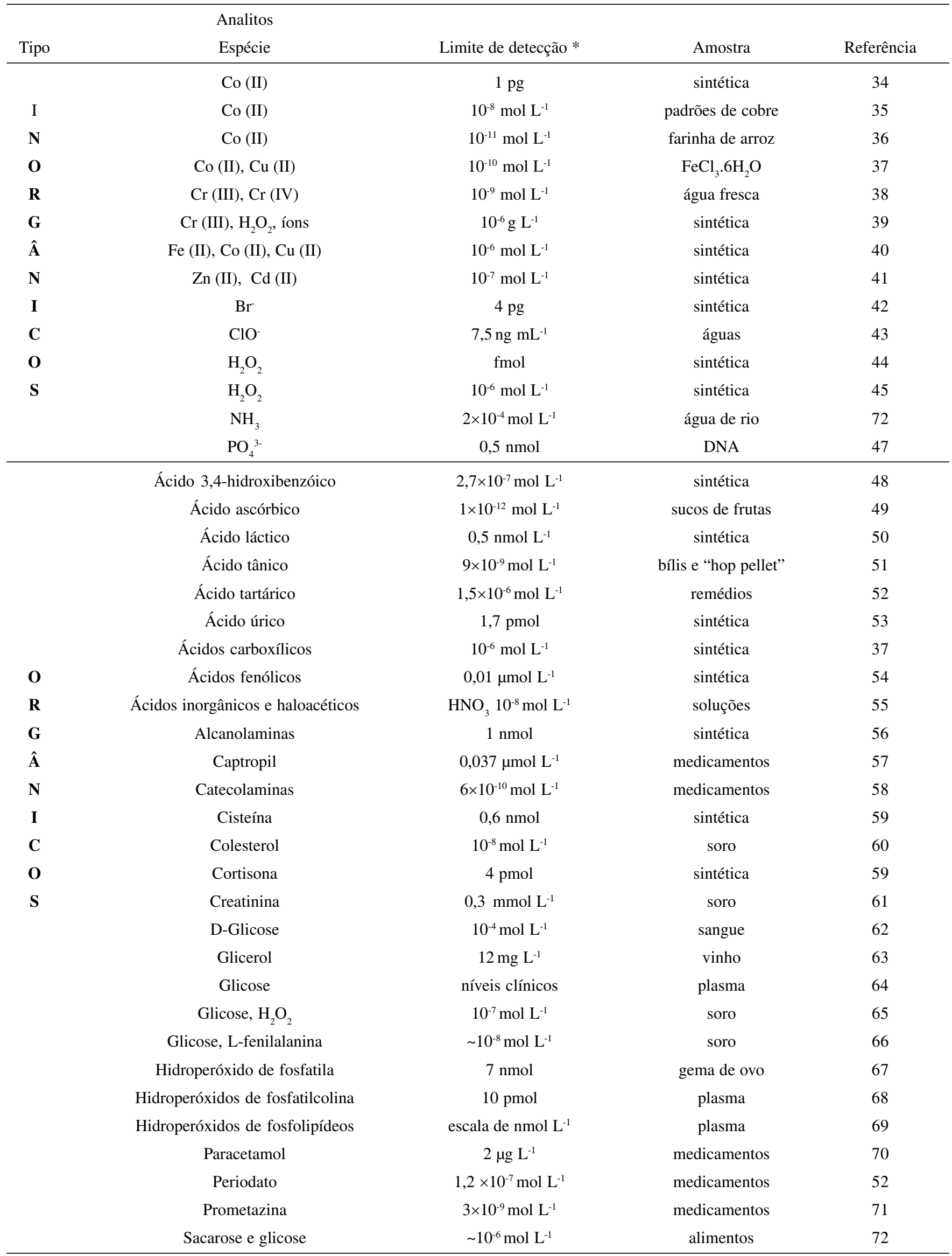

* Conforme definição nos artigos originais correspondentes 


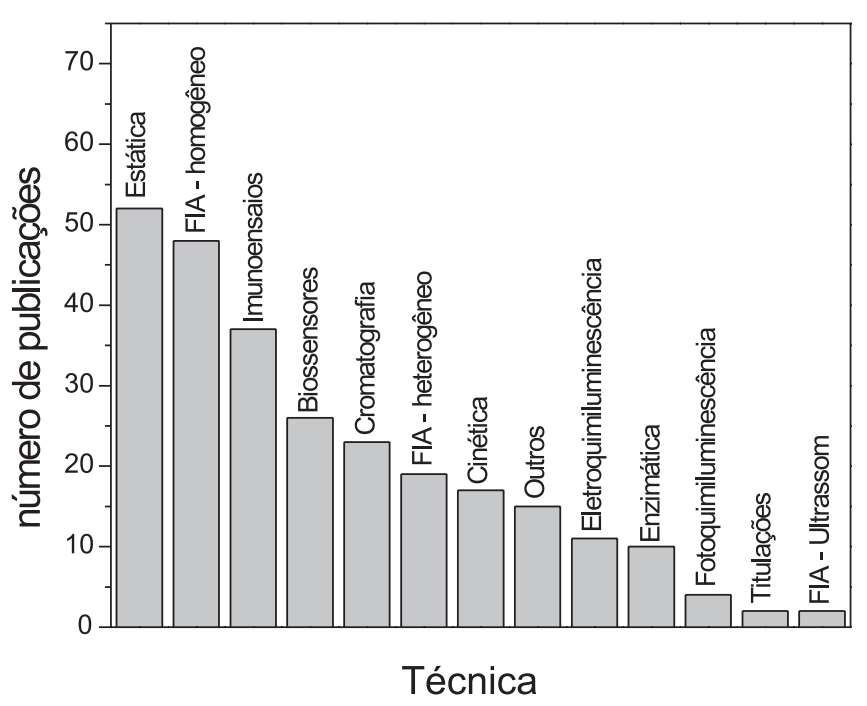

Figura 8. Aplicações analíticas da reação quimiluminescente do luminol divididas pelas técnicas utilizadas desde 1945

gica e ambiental, empregando-se a maioria das técnicas analíticas já consagradas.

Por outro lado, nota-se um número reduzido de trabalhos sobre a utilização da reação quimiluminescente do luminol em métodos cinéticos de análise com pouca participação brasileira, embora, por exemplo, estejam sendo desenvolvidos trabalhos nessa linha em nosso laboratório ${ }^{28,29,75-78}$. Considerando-se a versatilidade da reação do ponto de vista analítico e as características positivas dos métodos cinéticos de análise, vislumbra-se uma linha de estudo com elevada perspectiva de bons resultados, que merece ser desenvolvida e pode inspirar pesquisadores interessados.

Cabe aqui uma avaliação do ritmo de desenvolvimento dos métodos cinéticos de análise e os trabalhos recentemente publicados para fundamentar tal premissa.

\section{MÉTODOS CINÉTICOS DE ANÁLISE}

\section{Conceito}

Diversas técnicas e métodos analíticos são baseados em medidas de mudanças físicas, químicas e físico-químicas de um sistema envolvendo uma reação química, que se inicia com uma dada velocidade e tende ao equilíbrio.

Num sistema reacional, pode-se distinguir duas regiões: a dinâmica ou região cinética, na qual ocorre a variação na velocidade de reação, e a estática ou região de equilíbrio, na qual os processos envolvidos atingem o equilíbrio. Ambas as regiões apresentam grande potencial para aplicação analítica. Os métodos clássicos de análise, de equilíbrio ou termodinâmicos, como gravimetria, volumetria e a maioria dos métodos instrumentais, são aplicados às reações quando estas se completam ou atingem o equilíbrio. Nos métodos cinéticos, a aquisição do sinal analítico é feita em condições dinâmicas, enquanto as concentrações dos reagentes e produtos estão variando, antes que a reação atinja o equilíbrio ${ }^{25}$. Métodos analíticos que envolvem medidas da variação da velocidade de uma reação química pela ação de ativadores ou inibidores são também métodos cinéticos.

As grandes vantagens dos métodos cinéticos de análise, em relação aos métodos de equilíbrio, são a possibilidade de aplicação ana- lítica de um número muito maior de reações, tanto rápidas quanto lentas, e o baixo custo. Apesar disso, inicialmente, houve certa resistência para disseminação dos métodos cinéticos de análise, devido à consolidada confiança nos métodos clássicos e à relativa complexidade para o tratamento dos dados experimentais. Atualmente, as técnicas instrumentais são populares e os métodos cinéticos expandem suas possibilidades de aplicação, sendo que a popularização dos computadores superou qualquer alusão a eventuais dificuldades para tratamento dos dados experimentais ${ }^{25}$.

Convém destacar outros aspectos favoráveis dos métodos cinéticos, como a possibilidade de minimizar interferências, o menor tempo de análise e a facilidade para automação. Além disso, as reações catalisadas, incluindo as enzimáticas, apresentam limites de detecção tão baixos quanto técnicas mais sofisticadas e utilizadas comercialmente, como cromatografia líquida de alta eficiência e espectroscopia de absorção atômica.

Não se pode negar que os métodos cinéticos de análise apresentam aspectos problemáticos. A reprodutibilidade dos resultados envolve a necessidade do controle das condições que afetam a velocidade das reações como temperatura, $\mathrm{pH}$, força iônica, solventes e o processo de adição e mistura de reagentes ${ }^{13}$. Porém, um balanço geral de suas características apresenta um saldo bastante favorável que justifica estudos para desenvolver aplicações.

\section{Desenvolvimento de trabalhos e tendências}

Nas últimas décadas do século XX, o desenvolvimento e o aprimoramento de novas técnicas de aquisição e tratamento de dados analíticos, conjuntamente com a necessidade crescente de métodos de análise mais sensíveis e seletivos, abriram caminho para novas propostas analíticas destacando-se, dentre outras, os métodos cinéticos de análise. Isto pode ser verificado pelo aumento no número de publicações com este enfoque, identificadas em um levantamento bibliográfico realizado com o banco de dados WEB OF SCIENCE ${ }^{73}$, com as seguintes palavras chave: "(kinetic or kinetics) and (analysis or determination)".

Desde 1945, foram encontradas catalogadas cerca de 1500 publicações (artigos, notas e resumos de encontros) sobre aplicações analíticas da cinética de reações em obras de referência deste "site".

Com a popularização do microcomputador, surgiram várias análises catalíticas e não-catalíticas e, na década de 80 , os métodos cinéticos representaram expressiva parcela das publicações científicas em Química Analítica ${ }^{13}$. O desenvolvimento das técnicas dinâmicas de análise, como os métodos de injeção em fluxo, de fluxo contínuo e segmentado, decididamente influenciou o crescimento das aplicações dos métodos cinéticos em várias áreas, como em análises clínicas, nas últimas décadas do século $\mathrm{XX}^{25}$.

Avaliando-se a média de publicações anual sobre métodos cinéticos de análise, nota-se crescimento significativo a partir de 1945 , com o máximo em 1991, quando mais de 80 trabalhos foram publicados. Desde os anos 70, cerca de 40 trabalhos sobre métodos cinéticos de análise são publicados por ano.

É interessante notar a distribuição por país de origem dos trabalhos sobre métodos cinéticos de análise. Encontram-se publicações provenientes dos mais diversos países. Até 1980, Estados Unidos, a então União Soviética, Espanha e Alemanha apresentaram o maior número de publicações. No final do século XX, destacou-se a Espanha com o maior número de publicações nessa linha, reforçando a reconhecida produtividade deste país em Química Analítica. A partir de 1990, outros centros começaram a se destacar com a aplicação dos métodos cinéticos, principalmente Japão, China e Irã.

A diversificação da origem dos trabalhos publicados pode ser relacionada com o baixo custo das técnicas de medida da análise 
cinética, o que pode ter estimulado a disseminação do tema. Países como Brasil, Índia, Egito e muitos outros também passaram a desenvolver pesquisas sobre métodos cinéticos, o que contribuiu para a popularização da técnica internacionalmente. Esta distribuição pode refletir a acessibilidade dos métodos cinéticos de análise devido às facilidades de execução desses métodos, o que favorece sua popularização.

Atualmente, os métodos cinéticos de análise destacam-se como uma opção analítica extremamente sensível e barata. Em comparação aos métodos de equilíbrio, os métodos cinéticos de análise vêm se tornando populares por sua simplicidade, precisão, menor tempo de análise, diminuição do número de interferentes e facilidade para a automação ${ }^{25}$. Além disso, quando estes métodos são associados às técnicas quimiométricas para tratamentos de dados, tornam-se ainda mais versáteis, simplificando até procedimentos de análise simultânea de misturas que são de crescente interesse ${ }^{79,80}$.

Desde 1945, o desenvolvimento das aplicações dos métodos cinéticos de análise vem crescendo, acompanhando os avanços tecnológicos. Desde a última década do século XX, estes métodos podem ser considerados uma área da Química Analítica reconhecidamente amadurecida por suas aplicações e seu desenvolvimento. A partir de novas perspectivas tecnológicas, como a robótica e técnicas sofisticadas de tratamento de dados, Crouch ${ }^{81}$ indica tendências para estudos com métodos cinéticos de análise, que podem ser assim resumidas:

- aumento do uso da automação inteligente, ou seja, todas as condições de análise controladas por computador;

- crescimento da utilização de dados multidimensionais;

- desenvolvimento de técnicas sofisticadas de tratamento de dados;

- progresso nos métodos de compensação de erros;

- inovações dos procedimentos cinéticos com multicomponentes;

- expansão das aplicações da determinação cinética e

- aplicação das aproximações cinéticas para vários tipos de respostas dependentes do tempo.

\section{CONCLUSÃO}

A reação quimiluminescente do luminol apresenta propriedades muito interessantes para o desenvolvimento de aplicações analíticas e isso vem sendo explorado intensamente, desde 1960. Porém, há poucos trabalhos empregando esta reação em métodos cinéticos de análise. Uma avaliação crítica das características desses métodos demonstra vários aspectos positivos que estimulam estudos para disseminar sua aplicação com novos sistemas, com a reação do luminol. Há detalhes desafiadores, intrínsecos desta reação que demandam esforços para viabilizar sua aplicação favorável em métodos cinéticos de análise, o que deve ser aproveitado em novos trabalhos, com boas perspectivas de resultados satisfatórios.

Neste contexto, o desenvolvimento de métodos cinéticos de análise com reações quimiluminescentes parece ser promissor, principalmente considerando-se o aspecto de baixo custo da técnica e a sensibilidade da reação. Toda esta apresentação pretende servir para encorajar estudos nesta linha, na qual a produção científica nacional ainda é incipiente.

\section{AGRADECIMENTOS}

À Fundação de Amparo à Pesquisa do Estado de São Paulo, FAPESP, pelo apoio financeiro para este trabalho (96/09175-0 e 99/ 00022-5). Ao Prof. Dr. A. P. Chagas pela leitura do manuscrito e pelas valiosas sugestões.

\section{REFERÊNCIAS}

1. Campbell, A. K.; Chemiluminescence: Principles and Applications in Biology and Medicine, Ellis Horwood Ltd.: Chichester, 1988.

2. Radziszewski, B.; Ber. Chem. Ges. 1877, 10, 70.

3. Wiedemann, E.; Ann. Physik \& Chemie 1888, 34, 446.

4. Albertin, R.; Arribas, M. A. G.; Bastos, E. L.; Röpke, E.; Sakai, P. N.; Sanches, A. M. M.; Stevani, C. V.; Umezu, I. S.; Yu, J.; Baader, W. J.; Quim. Nova 1998, 21, 772.

5. Robards, K.; Worsfold, P. J.; Anal. Chim. Acta 1992, 266, 147.

6. Nery, A. L. P.; Baader, W. J.; Quim. Nova 2001, 24, 626.

7. Lee, J.; Cormier, M. J.; Hercules, D. M. Em Chemiluminescence and Bioluminescence; Cormier, M. J.; Hercules, D. M.; Lee, J., eds.; Plenum Press: New York, 1973, p. 1

8. Gundermann, K. D.; Mccapra, F.; Chemiluminescence in Organic Chemistry, Springer-Verlag: Berlin, 1987.

9. Kellner, R.; Mermet, J.-M.; Otto, M.; Widmer, H. M.; Analytical Chemistry: the approved text to the FECS curriculum analytical chemistry, Wiley-VCH Verlag GmbH: Weinheim, 1998.

10. Worsfold, P. J.; Anal. Chim. Acta 1992, 266, 145.

11. Jiménez, A. M.; Navas, M. J.; Crit. Rev. Anal. Chem. 1997, 27, 291.

12. Navas, M. J.; Jiménez, A. M.; Crit. Rev. Anal. Chem. 2000, 30, 153.

13. Mottola, H. A.; Kinetics Aspects of Analytical Chemistry, John Wiley \& Sons: New York, 1988.

14. Albrecht, H. O.; Z. Phys. Chem. 1928, 10, 70.

15. Merényi, G.; Lind, J.; Eriksen, T. E.; J. Biolum. Chemilum. 1990, 5, 53.

16. Eriksen, T. E.; Lind, J.; Merényi, G.; J. Chem. Soc. Faraday Trans. 1 1981, 77, 2125.

17. Ljunggren, S.; Merényi, G.; Lind, J.; J. Am. Chem. Soc. 1983, 105, 7662.

18. Merényi, G.; Lind, J.; Eriksen, T. E.; J. Phys. Chem. 1984, 88, 2320.

19. Merényi, G.; Lind, J.; Eriksen, T. E.; J. Am. Chem. Soc. 1986, 108, 7716.

20. Merényi, G.; Lind, J.; Eriksen, T. E.; J. Phys. Chem. 1990, 94, 748.

21. Lind, J.; Merényi, G.; Eriksen, T. E.; J. Am. Chem. Soc. 1983, 105, 7655.

22. Merényi, G.; Lind, J.; $5^{\text {th }}$ Symphosium on Radiation Chemistry, Budapeste, Hungria, 1983.

23. Michl, J.; Photochem. Photobiol. 1977, 25, 141.

24. Xiao, C. B.; King, D. W.; Palmer, D. A.; Wesolowski, D. J.; Anal. Chim. Acta 2000, 415, 209.

25. Perez-Bendito, D.; Silva, M.; Kinetic Methods in Analytical Chemistry, Ellis Horwood Ltd: Chichester, 1988.

26. Salem, A.; Salem, M. A.; Gemeay, A. H.; J. Mol. Catal. 1993, 84, 67.

27. Zhou, H.; Shen, Y. F.; Wang, J. Y.; Chen, X.; O’Young C.-L.; Suib, S. L.; J. Catal. 1998, 176, 321.

28. Ferreira, E. C.; Rossi, A. V.; Resumos da 23a Reunião Anual da Sociedade Brasileira de Química, Poços de Caldas, Brasil, 2000.

29. Ferreira, E. C.; Dissertação de Mestrado, Universidade Estadual de Campinas, Brasil, 2001.

30. Preuschoff, F.; Spohn, U.; Weber, E.; Unverhau, K.; Mohr, K. H.; Anal. Chim. Acta 1993, 280, 185.

31. Sekine, Y.; Suzuki, M.; Takeuchi, T.; Tamiya, E.; Karube, I.; Anal. Chim. Acta 1993, 280, 179.

32. Ci, Y.-X.; Zheng, Y.-G.; Tie, J.-K.; Chang, W.-B.; Anal. Chim. Acta 1993, 282, 695 .

33. Kamidate, T.; Ishikawa, A.; Watanabe, H.; Abe, S.; Bull. Chem. Soc. Jpn. 1993, 66, 655

34. Burguera, J. L.; Towsend, A.; Greenfield, S.; Anal. Chim. Acta 1980, 114, 219

35. Sakai, H.; Fujiwara, T.; Yamamoto, M.; Kumamamru, T.; Anal. Chim. Acta 1989, 221, 249.

36. Jones, P.; Williams, T.; Ebdon, L.; Anal. Chim. Acta 1989, 217, 157.

37. Yan, B.; Lewis, S. W.; Worsfold, P. J.; Lancaster, J.S.; Gachanja, A.; Anal. Chim. Acta 1991, 250, 145.

38. Williams, T.; Jones, P.; Ebdon, L.; J. Chromatogr. 1989, 482, 361.

39. Yan, B.; Worsfold, P. J.; Robards, K.; Analyst 1991, 116, 1227.

40. Klopf, L. L.; Nieman, T. A.; Anal. Chem. 1983, 55, 1080.

41. Burguera, J. L.; Burguera, M.;Towsend, A.; Anal. Chim. Acta 1981, 127, 199.

42. Shakir, I. M. A.; Faizullah, A. T.; Analyst 1989, 114, 951.

43. Gonzales-Robledo, D.; Silva, M.; Perez-Bendito, D.; Anal. Chim. Acta 1990, 228, 123.

44. Nabi, A.; Worsfold, P. J.; J. Chem. Soc. Pak. 1987, 9, 575.

45. Eremim, S. A.; Vlasenko, S. B.; Osipov, A. P.; Eremina; I. D.; Egerov, A. M.; Anal. Lett. 1989, 22, 2037.

46. Kraus, P. R.; Crouch, S. R.; Anal. Lett. 1987, $20,183$.

47. Kawasaki, H.; Sato, K.; Ogawa, J.; Hasegawa, Y.; Yuki, H.; Anal. Biochem. 1989, 182, 336. 
48. Cui, H.; Jiang, H.; Meng, R.; He, C.; Zhao, H.; Spectrosc. Spect. Anal. 1999, 19, 377.

49. Alwarthan, A. A.; Analyst 1993, 118, 639

50. Busch, M.; Polster, J.; Trends Anal. Chem. 1992, 11, 230.

51. Cui, H.; Li, Q.; Meng, R.; Zhao, H.; He, C.; Anal. Chim. Acta 1998, 362, 151.

52. Gaikwad, A.; Silva, M.; Perez-Bendito, D.; Analyst 1994, 119, 1819.

53. Feng, M. L.; Huang, Y. W.; Zhang, Z. J.; Acta Chim. Sinica 1997, 55, 806.

54. Diaz, A. N.; Garcia, J. A. G.; Anal. Chem. 1994, 66, 988.

55. Shakir, I. M. A.; Faizullah, A. T.; Analyst 1990, 115, 69.

56. Worsfold, P. J.; Yan, B.; Anal. Chim. Acta 1991, $246,447$.

57. Xinrong, Z.; Baeyens, W. R. G.; VanderWeken, G.; Calokerinos, A. C. Nakashima, K.; J. Pharm. Biomed. Anal. 1995, 13, 425.

58. Zhang, C. X.; Huang, J. C.; Zhang, Z. J.; Aizawa, M.S.; Anal. Chim. Acta 1998, 374, 105 .

59. Kubo, H.; Toriba, A.; Anal. Chim. Acta 1997, 353, 345.

60. Blum, L. J.; Plaza, J. M.; Coulet, P. R.; Anal. Lett. 1987, 20, 317.

61. Petersson, B. A.; Hansen, E. H.; Ruzicka, J.; Anal. Lett. 1986, 19, 649.

62. Petersson, B. A.; Anal. Lett. 1989, 22, 83.

63. Puchades, R.; Lemieux, L.; Simard, R. E.; J. Food Sci. 1991, 56, 1097.

64. Worsfold, P. J.; Farrely, J.; Matharu, M. S.; Anal. Chim. Acta 1984, 164, 103.

65. Abdel-Latif, M. S.; Guibault, G. G.; Anal. Chim. Acta 1989, 221, 11.
66. Igarashi, S.; Hinze, W. L.; Anal. Chim. Acta 1989, 225, 147.

67. Miyazawa, T.; Yasuda, K.; Fujimoto, K.; Anal. Lett. 1987, 20, 915.

68. Miyazawa, T.; Saeki, R.; Inaba, H.; J. Biolumin. Chemilumin. 1989, 4, 475.

69. Miyazawa, T.; Fujimoto, K.; Oikawa, S.; Biomed. Chromatogr. 1990, 4, 131

70. Alapont, A. G.; Zamora, L. L.; Calatayud, J. M.; J. Pharm. Biomed. Anal. 1999, 21, 311 .

71. Alwarthan, A. A.; Al-Tamrah, S. A.; Akel, A. A.; Anal. Chim. Acta 1993, $282,169$.

72. Koerner, C. A.; Nieman, T. A.; Anal. Chem. 1986, 58, 116

73. Kamidate, T.; Kuniya, I.; Watanabe, H.; Chem. Lett. 1993, 12, 2049.

74. http://webofscience.fapesp.br, acessada em Outubro 2001.

75. Lebedeva, O. V.; Ugarova, N. N.; J. Anal. Chem. 1995, 50, 1017.

76. Ferreira, E. C.; Rossi, A. V.; Resumos da $24^{a}$ Reunião Anual da Sociedade Brasileira de Química, Poços de Caldas, Brasil, 2001.

77. Ferreira, E. C.; Rossi, A. V.; Rohwedder, J. J. R.; Resumos da $24^{a}$ Reunião Anual da Sociedade Brasileira de Química, Poços de Caldas, Brasil, 2001.

78. Ferreira, E. C.; Rossi, A. V.; trabalho não publicado.

79. Xie, Y.; Baeza-Baeza, J. J.; Ramis-Ramos, G. A; Anal. Chim. Acta 1996, 321,75 .

80. Cueva, J. M. D.; Rossi, A. V.; Poppi, R. J.; Chem. Intell. Lab. Sys. 2001, $55,125$.

81. Crouch, S. R.; Anal. Chim. Acta 1993, 283, 453. 